\title{
Distressed personality is associated with lower psychological well-being and life satisfaction, but not disability or disease activity in rheumatoid arthritis patients
}

\author{
Kim Klaassen • Ivan Nyklíček • Simone Traa • Ron de Nijs
}

Received: 18 April 2011 /Revised: 5 December 2011 / Accepted: 5 December 2011 / Published online: 22 December 2011

(C) The Author(s) 2011. This article is published with open access at Springerlink.com

\begin{abstract}
The distressed personality type ("type D personality") has been shown to be associated with low quality of life and higher morbidity and mortality in various patient groups. Because the role of type D personality is unknown in patients with rheumatoid arthritis (RA), the aim of the present study was to investigate the association of type D personality with aspects of quality of life and disease activity in RA patients. In addition, a potential buffering effect by accepting mindfulness was examined. Participants were 147 patients between 22 and 87 years of age. Patients completed relevant questionnaires at home and the disease activity score was determined. After controlling for potentially confounding variables, multivariate analyses of covariance showed an association of type D personality with a lower satisfaction with life $(p<0.001)$ and a lower psychological well-being $(p<0.001)$, but not disease activity in RA patients. Although mindfulness was associated with a higher satisfaction with life $(p=0.02)$ and positive $\operatorname{mood}(p=0.01)$, it did not diminish the unfavourable associations between type D and well-being. In conclusion, although type D personality is related with lower well-being, it does not seem to be associated with disability or disease activity in RA patients.
\end{abstract}

K. Klaassen • I. Nyklíček ( $\bowtie)$

Center of Research on Psychology in Somatic disease (CoRPS),

Department of Medical Psychology, Tilburg University,

PO Box 90153, 5000 LE, Tilburg, The Netherlands

e-mail: i.nyklicek@tilburguniversity.edu

S. Traa

Department of Medical Psychology, Máxima Medical Centre, Eindhoven, The Netherlands

R. de Nijs

Regional Rheumatic Center ZO-Brabant,

Eindhoven, The Netherlands
Keywords Disease activity · Distressed (type D) personality $\cdot$ Mindfulness $\cdot$ Quality of life $\cdot$ Rheumatoid arthritis · Well-being

\section{Introduction}

Rheumatoid arthritis (RA) is the most common chronic autoimmune arthritis $[1,2]$. The disease generally follows a remitting-flaring course of inflammation and pain and leads to increasing functional disability $[1,2]$. Patients confronted with the diagnosis of RA have to change their way of living [3] and they experience psychological distress more often than people in the general population [4]. The disease requires adaptation and involves many future uncertainties because it cannot be cured. Therefore quality of life in this population is recognised as a very important treatment outcome [5]. The evaluation of this quality depends not only on the disease activity. Individuals can vary greatly in their psychological adaptation to the disease and this is not always explained by variations in inflammatory activity [6]. Patients with a comparable clinical picture and time since their diagnosis can evaluate their quality of life quite differently. This means that other factors than only the medical parameters may be responsible for these differences. An emerging area of explanation of individual differences in quality of life and physical functioning in medical patients in general involve relatively stable individual dispositions such as personality [7].

There is only little research regarding the relation between personality and the individual differences in quality of life and physical functioning in rheumatoid arthritis patients. Optimism and the tendency to hold greater positive outcome expectancies have been found to be related to better psychological well-being in RA patients [8]. 
Optimism may have a positive impact on physical health as well, optimistic patients report fewer symptoms [9]. Patients with negative affectivity (the tendency to experience negative mood states across situations and one of the components of type D personality, see below) are reported to show lower health satisfaction scores [10]. RA patients in general report higher levels of negative affectivity compared to the general population $[3,11]$. Negative affectivity is associated with more negative emotions, including more symptoms of anxiety and depression, which are known to affect quality of life unfavourably [12].

Psychological characteristics have been found to be important determinants of functioning in other chronic diseases. The distressed personality type (type D personality) has been identified as an important explanatory factor of individual differences in quality of life and also clinical outcomes in various patient groups, ranging from chronic pain patients to patients with malignant melanoma [13]. Type D personality refers to patients who frequently experience negative emotions across situations (are high in negative affectivity) in combination with a tendency not to express affect to others (termed social inhibition) [7, 14, 15]. Most type D personality research has been performed in patients with cardiovascular diseases [7, 16], in which patients with a type D personality have been found not only to have an increased risk of psychological distress, but also adverse clinical health status [7, 14, 17] and impaired quality of life [18]. Type D personality is also associated with a high risk of cardiac events, such as myocardial infarction $[15,19]$ and a threefold increased risk of a cardiac death in cardiac patients $[14,17]$. In addition, type D personality has been found to be associated with enhanced levels of proinflammatory cytokines TNF- $\alpha$ and its receptors [20], which are important disease parameters in RA [21].

While the role of type D personality in cardiac and some other somatic patients has been found, the role of this psychological characteristic in rheumatoid arthritis patients is unknown. Given its relations with psychological wellbeing, quality of life and levels of pro-inflammatory cytokines in other patient samples, type D personality may be an important factor in rheumatoid patients as well.

In addition, mindfulness may be hypothesised to be a buffering factor concerning the negative consequences of type D personality on aspects of quality of life and perhaps even physical health status. Mindfulness is commonly defined as the state of being attentive to and aware of what is taking place in the present with an open and accepting (nonjudgemental) attitude. This quality of attention to the present moment has been claimed to buffer adverse consequences of negative emotions, which are strongly present in type Ds, by (a) identification and letting go of ruminative thoughts about the past or future and (b) mental and bodily relaxation [22]. Indeed, mindfulness-based psychological interventions have been reported to enhance quality of life [23-25] and decrease pain or coping with pain $[26,27]$ in various clinical and non-clinical samples. In addition, preliminary data suggest that mindfulness is associated with psychological wellbeing in fibromyalgia patients [28] and that mindfulnessbased interventions may decrease $\mathrm{T}$ cell production of proinflammatory cytokines [29] which may be beneficial in RA patients.

Therefore, the aim of the study was to investigate the association of the distressed personality type (type D personality) with aspects of quality of life, including psychological well-being, life satisfaction and disability, as well as disease activity in rheumatoid arthritis patients and the potentially buffering effect of mindfulness. The hypotheses are that (a) type D patients report lower levels of these aspects of quality of life compared with non-type D patients, while (b) they show a more severe disease activity; (c) mindfulness buffers the effects mentioned in hypotheses (a) and (b).

\section{Method}

Patients and procedure

Participants were recruited in the Regional Rheumatic Center ZO-Brabant, Eindhoven, the Netherlands, and were provided with information about the study by the rheumatologist. The study complied with the Helsinki Declaration on human subjects; the protocol being approved by the Medical Ethics Committee of the Máxima Medical Center, Eindhoven, the Netherlands.

Inclusion criteria were a diagnosis of rheumatoid arthritis, starting treatment with methotrexate or a TNF- $\alpha$ antagonist (for a substudy not reported on here) and sufficient understanding of written and spoken Dutch language. All patients had to be included within 4 weeks after start with this medication. The exclusion criteria were age $\geq 90$ years, chronic severe psychiatric conditions (e.g. psychosis or a personality disorder) and any infections during last week. If the latter condition was the case, but was very temporary (such as common cold), we aimed to postpone the inclusion by 1 or 2 weeks, depending on the duration of the condition.

After explanation of the study, patients were asked to complete a questionnaire at home and to send it back within 1 week. In the case of refusal, a short questionnaire with general variables such as gender, age, marital status, level of education and the time since diagnosis was attached to be able to compare participants with nonparticipants. Data were collected from 162 individuals: 147 participants (91\%) who completed questionnaires and 15 nonparticipants $(9 \%)$. None of the patients refused to provide the general data. 
The disease activity score was measured by the rheumatologist and had to be determined within a month before and a month after the questionnaire was completed. Average time between patients' completion of the questionnaire and measurement of disease activity score was 5.6 \pm 9.6 days.

\section{Materials}

\section{Questionnaires}

Type D personality was measured using the 14-item type D Scale (DS14) [15]. This questionnaire consists of two subscales, negative affectivity and social inhibition, of seven items each. Items are scored on a five-point Likert scale ranging from 0 (false) to 4 (true). A cutoff score of $\geq 10$ on both scales is conventionally used to classify subjects as having type D personality. In previous research on the Dutch version, the test-retest reliability over a 3-month period was 0.72 and 0.82 for negative affectivity and social inhibition, respectively, and the internal consistency (Cronbach's $\alpha$ ) was 0.88 and 0.86 , respectively. Evidence for construct validity is also provided [15]. In this sample, the internal consistency was 0.83 for both subscales.

Mindfulness was assessed by means of the Freiburg Mindfulness Inventory-short-form (FMI-s) [30]. The FMI$\mathrm{s}$ consists of 14 items and is regarded as an index of overall mindfulness, including the aspects of attention to external and internal (proprioceptive and psychological) phenomena in the present moment, accepting self-reflection and a nonreacting stance. These items are scored on a four-point Likert scale ranging from 1 (rarely) to 4 (almost always). The original FMI-s has shown good construct validity and internal consistency (Cronbach's $\alpha$ of 0.86) [30]. Because no Dutch translation was available at the start of the study, the second author (IN) translated the FMI-s into Dutch together with Drs. M. van der Lee and C. Völker (Helen Dowling Institute, the Netherlands) and Dr. M. Schroevers (Groningen University, Netherlands), one of which is a native German speaker, using the standard back-translation method. Because of this new translation, we carefully tested the internal consistency in the present sample, resulting in a satisfactory Cronbach's $\alpha$ of 0.79 .

Various instruments were used to measure different aspects of quality of life. The Health Assessment Questionnaire [31] has been validated in groups of patients with a wide variety of rheumatic diseases, including rheumatoid arthritis, and measures the degree of disability. We used the standard Dutch version [32], which consists of 20 items in eight categories which represent a comprehensive set of functional activities (dressing and grooming, arising, eating, walking, hygiene, reach, grip and common daily activities). These questions are part of the disability index score, without including questions regarding aids and devices and ask about the past week: "Are you able to..." perform a particular task. The responses are scored on a four-point Likert scale ranging from 0 (without any difficulty) to 3 (unable to do) [31]. The Dutch version has good reliability: Cronbach's $\alpha$ was between 0.85 and 0.95 in previous research [32] and 0.89 in this sample.

The Dutch version of the Satisfaction with Life Scale [33] is a brief questionnaire consisting of five items using a seven-point Likert scale ranging from 1 (strongly disagree) to 7 (strongly agree). It measures how satisfied patients are with their present life. This questionnaire has an adequate construct validity and internal consistency: Cronbach's $\alpha$ of 0.87 in previous research [33] and 0.92 in this study.

The Dutch Global Mood Scale [34] consists of ten positive and ten negative mood terms. The patients have to answer on a five-point Likert scale ranging from 0 (not at all) to 4 (extremely), the extent to which they have experienced each mood state lately. This questionnaire has adequate construct validity and good internal consistency (Cronbach's $\alpha$ of 0.90 in previous research) [34] and Cronbach's $\alpha$ of 0.93 and 0.88 for negative and positive affect, respectively in this study.

The Dutch Symptoms of Anxiety and Depression [35] scale consists of four items which are scored on a five-point Likert scale ranging from 0 (not at all) to 4 (very much). The questionnaire is a reliable and brief index of mixed anxietydepression symptoms. In this sample the Cronbach's $\alpha$ was 0.85 . Also in previous research, the scale had good internal consistency (Cronbach's $\alpha$ of 0.86) and validity [35].

Finally a questionnaire was employed for assessing different sociodemographic control variables, such as age, gender, education level (low =lower than midlevel vocational education; high is at least midlevel vocational education) and marital status (having a partner or not). The medical control variables time since diagnosis and treatment (methotrexate or a TNF- $\alpha$ antagonist) were obtained from medical records.

\section{Disease activity score}

The Disease Activity Score (DAS-28) [2] is originally a Dutch instrument that indicates how active the RA is at a certain moment. The DAS-28 consists of four parts: (a) the amount of swollen joints and (b) the amount of tender joints as determined by the rheumatologist, (c) a general health assessment on a visual analogue scale (VAS) (from 1-100) obtained from the patient on self-evaluated disease severity and (d) erythrocyte sedimentation in the blood. The DAS-28 score can be calculated using the formula; DAS-28 $=0.54(\sqrt{ }$ number of tender joints) +0.065 (number of swollen joints) + 0.33 (Ln erythrocyte sedimentation) +0.0072 (VAS score). This total score is a number between 0 and 10, indicating the amount of disease severity. This composite measure has shown adequate validity $[2,36]$. 
Statistical analysis

All analyses were performed using SPSS software. First participants and nonparticipants were compared on age, gender, education, marital status and time since diagnosis using independent sample $t$ tests or chi-square tests.

The main analyses were multivariate analyses of covariance (MANCOVA) to examine associations between type D personality (independent variable) and the outcome variables. Separate analyses were performed for the cluster of psychological variables (negative affect, positive affect, symptoms of anxiety and depression, health status and satisfaction with life) and for the cluster of disease activity variables (swollen joints, tender joints, level of erythrocyte sedimentation and VAS). In addition, the association between type D, mindfulness and total DAS-28 was examined using a separate univariate analysis of covariance. Finally, the type D by mindfulness interaction was explored on all outcome variables to examine the potential buffer effect by mindfulness. An $\alpha$ level of 0.05 was used in all analyses. In addition, all analyses were controlled for age, gender, education level, time since diagnosis and treatment condition (methotrexate versus TNF- $\alpha$ ).

\section{Results}

Participants had a higher educational level than individuals who did not participate in the study: $87(59.6 \%)$ versus 3 $(20.0 \%)$ patients had at least midlevel vocational education $\left(\chi^{2}[1]=7.12, p=0.008\right)$. The nonparticipants did not differ from participants (all $p>0.10)$ regarding age $(57.4 \pm 11.3 \mathrm{vs}$ $57.2 \pm 10.7$ years), gender (81 (55.1\%) vs $10(66.7 \%)$ women), marital status (117 (80.1\%) vs $11(73.3 \%)$ having a partner), treatment (116 (78.9\%) vs $10(66.7 \%)$ having methotrexate) or time since diagnosis $(51.6 \pm 101.6$ vs $42.4 \pm$ 54.7 months).

Due to occasional missing data on the questionnaire data, analyses on questionnaire data were based on 141 patients, of whom $43(30.5 \%)$ had a type D personality and 98 (69.5\%) were not type Ds, which is comparable to previous findings in cardiac patients [15]. Patients with a type D personality did not differ from non-type Ds regarding all sociodemographic and background medical variables (Table 1). Type D patients did score lower on mindfulness compared with non-type D patients $(35.0 \pm 5.7$ vs $38.4 \pm 6.5 ; t$ $[139]=2.94, p=0.004)$.

Aspects of quality of life

Several covariates showed significant associations with some aspects of quality of life. Age was negatively associated with negative affect $(F[1,124]=6.56, p=0.01$; unstandardized $\beta$ coefficient $\left.=-0.20, \eta^{2}=0.05\right)$, positively with life satisfaction $(F[1,124]=6.33, p=0.01$, unstandardized $\left.\beta=0.15, \eta^{2}=0.05\right)$, but also positively with disability ratings $(F[1,124]=4.09, p=0.05$, unstandardized $\beta=0.01$, $\left.\eta^{2}=0.03\right)$. Women reported a higher disability than men $(F$ $\left.[1,124]=5.99, p=0.02, \eta^{2}=0.05\right)$ and reported more symptoms of anxiety and depression $(F[1,124]=8.66, p=0.01$, $\left.\eta^{2}=0.07\right)$. Longer time since diagnosis was associated with higher disability scores $(F[1,124]=6.67, p=0.01$, unstandardized $\left.\beta=0.002, \eta^{2}=0.05\right)$, but fewer symptoms of anxiety and depression $(F[1,124]=4.19, p=0.04$, unstandardized $\left.\beta=-0.01, \eta^{2}=0.03\right)$. There were no significant associations between educational level and treatment condition and wellbeing or quality of life $(p>0.10)$.

Type D patients differed significantly from non-type D patients on the omnibus test of the combined dependent variables of aspects of quality of life $(F[5,118]=7.54, p<$ $\left.0.001, \eta^{2}=0.24\right)$, while controlled for age, sex, education, treatment and time since diagnosis. Patients with a type D personality reported a lower psychological well-being than non-type $\mathrm{D}$ patients. They showed more symptoms of anxiety and depression $\left(F[1,122]=17.37, p<0.001, \eta^{2}=0.13\right)$, more negative affect $\left(F[1,122]=6.52, p=0.01, \eta^{2}=0.05\right)$ and less positive affect $\left(F[1,122]=9.10, p=0.003, \eta^{2}=\right.$ 0.07) (Table 2). Type D patients also reported a lower satisfaction with life than non-type $\mathrm{D}$ patients $(F[1,122]=$ 25.44, $p<0.001, \eta^{2}=0.17$ ), but there was no significant difference regarding the disability score $(F[1,122]=0.34$, $p=0.56, \eta^{2}=0.003$ ).

When mindfulness was added to the model, the interaction effect of type $\mathrm{D}$ by mindfulness was not significant in the omnibus test $\left(F[5,117]=0.86, p=0.51, \eta^{2}=0.01\right)$ or in univariate analyses (all $p>0.10$ ). Mindfulness did show a significant omnibus main effect on the combined dependent variables of aspects of quality of life $(F[5,117]=2.71, p=$ $\left.0.02, \eta^{2}=0.11\right)$. Univariate analyses showed that mindfulness was positively associated with positive affect ( $F$ $[1,121]=9.14, p=0.003$; unstandardized $\beta$ coefficient $=$ $\left.0.35, \eta^{2}=0.05\right)$ and satisfaction with life $(F[1,121]=5.58$, $p=0.02$; unstandardized $\beta=0.24, \eta^{2}=0.05$ ), but no associations were found with the other variables.

\section{Disease activity}

Due to 42 missing values on the disease activity score, the number of participants in the analyses on disease activity was limited to 105 . None of the covariates showed a significant association with disease activity (all $p>0.10$ ). Type D patients did not differ from non-type $\mathrm{D}$ patients on the combined dependent variables of disease activity in the omnibus test $\left(F[4,100]=0.81, p=0.52, \eta^{2}=0.02\right)$. Also in univariate tests on the separate DAS components and total DAS-28, no effects were obtained on erythrocyte sedimentation, 
Table 1 Characteristics of patients with rheumatoid arthritis with and without distressed (type D) personality: means (SD) or numbers (\%)

Higher education - at least midlevel vocational education ${ }^{*} p<0.01$

\begin{tabular}{lccc}
\hline & Type D personality $(N=43)$ & Non-type D personality $(N=98)$ & t or $\chi^{2}$ \\
\hline Age & $54.9(11.2)$ & $58.4(11.3)$ & 1.70 \\
Higher education & $27(62.8 \%)$ & $59(60.2 \%)$ & 0.01 \\
Female sex & $25(58.1 \%)$ & $54(55.1 \%)$ & 0.00 \\
Having a partner & $32(74.4 \%)$ & $83(84.7 \%)$ & 0.75 \\
TNF-alpha antagonist & $7(16.3 \%)$ & $23(23.5 \%)$ & 0.69 \\
Months since diagnosis & $33.3(72.3)$ & $60.0(111.7)$ & 1.68 \\
Mindfulness & $35.0(5.7)$ & $38.4(6.5)$ & $2.94^{*}$ \\
\hline
\end{tabular}

swollen joints, tender joints, VAS and total DAS-28 (all $p>$ $0.10)$. Also mindfulness and the type $\mathrm{D}$ by mindfulness interaction did not show significant effects: omnibus test $(F[4$, $93]=0.38, p=0.82, \eta^{2}=0.02$ and $F[4,93]=1.45, p=0.22, \eta^{2}=$ 0.06 , resp.) and univariate tests $(p>0.10)$, except for erythrocyte sedimentation $\left(F[1,96]=4.00, p=0.048, \eta^{2}=0.04\right)$. Posthoc analyses revealed a nonsignificant tendency for mindfulness to be associated with lower erythrocyte sedimentation in type $\mathrm{D}$, but not in non-type $\mathrm{D}$, individuals $(r=-0.19, N=32$ type $\mathrm{D}, p>0.10$ versus $r=0.11, N=78$ non-type $\mathrm{D}, p>0.10)$.

\section{Discussion}

The main aim of the present study was to examine the association of the distressed (type D) personality with aspects of quality of life, including psychological wellbeing, life satisfaction, and disability, as well as the disease activity in RA patients. In multivariate analyses, in which various potentially confounding variables were controlled, type D patients reported lower positive mood, higher negative affect, more symptoms of anxiety and depression and lower general satisfaction with life compared to non-type D patients. No associations were found between type D personality and disability or disease activity scores. Also no buffering effects by mindfulness were obtained. Although the interaction effect between type $\mathrm{D}$ and mindfulness on erythrocyte sedimentation was in the expected direction and significant, the simple effects within type D and non-type D groups were not. Because this may have been due to the small subgroup sample sizes as a result of missing sedimentation values, it may be fruitful for future studies to examine this interaction effect in a larger sample. All in all, these present results suggest that type D personality may be an important psychological characteristic associated with psychological well-being and life satisfaction, but not with reported disability or objective disease indicators, in RA patients.

The association between type D and lower psychological well-being and aspects of quality of life has been found earlier in several studies in cardiovascular patients [7, 18]. In these patients, a relation was also found between type $\mathrm{D}$ personality and enhanced levels of pro-inflammatory cytokines [20] and self-evaluated cardiac symptoms [15, 18]. The association between type D personality and disease activity was not found in the present sample of RA patients.

First, one should note that in contrast to cardiovascular patients, all RA patients use some form of anti-inflammatory medication. Nevertheless, the lack of associations with reported disability and objective disease indicators may be
Table 2 Type D personality in relation to psychological well-being, quality of life and disease activity: means (and standard errors) from a MANCOVA analysis

$V A S$ visual analogue scale of self-perceived disease severity, $D A S-28$ disease activity score-28

$* p<0.05 ; * * p<0.01$

${ }^{a}$ The higher $N$ concerns self-report data, the lower $N$ concerns disease activity; analyses were controlled for age, gender, education level, time since diagnosis and treatment condition (methotrexate or TNF $\alpha$ antagonist)

\begin{tabular}{lcc}
\hline & Type D $(N=31 / 43)^{\mathrm{a}}$ & Non-type D $(N=74 / 98)^{\mathrm{a}}$ \\
\hline Quality of life & & \\
Life satisfaction & $20.94(1.00)^{*}$ & $27.12(0.68)^{* *}$ \\
Disability score & $1.13(0.10)$ & $1.06(0.07)$ \\
Psychological well-being & & \\
Positive affect & $16.91(1.22)^{*}$ & $21.38(0.82)^{* *}$ \\
Negative affect & $16.72(1.43)^{* *}$ & $12.26(0.97)^{*}$ \\
Anxiety and depression & $5.56(0.48)^{*}$ & $3.12(0.33)^{* *}$ \\
Disease activity & & \\
Swollen joints & $6.09(0.94)$ & $7.08(0.61)$ \\
Tender joints & $5.53(0.86)$ & $6.61(0.56)$ \\
VAS & $55.96(4.81)$ & $50.27(3.13)$ \\
Erythrocyte sedimentation & $27.88(4.06)$ & $28.43(2.64)$ \\
Total DAS-28 & $4.77(0.25)$ & $4.70(0.16)$ \\
\hline
\end{tabular}


due to the fact that these involve either ratings of ability to perform rather concrete and specific behaviours - in case of the disability index - or mainly ratings by rheumatologists in combination with physiological parameters, both of which are less or not at all influenced by the subjective interpretation by the patient as compared to the psychological variables used in this study. It is known that type D personality is especially associated with a gloomy view of life, as reflected by associations with all kinds of psychological symptoms and low ratings of subjective satisfaction with quality of life [13, 18].

Why mindfulness did not buffer these associations is difficult to explain. One possibility is the fact that patients with a type D personality scored significantly lower on mindfulness compared to non-type Ds. Perhaps their lower mindfulness scores prevented mindfulness to be able to buffer any effects on psychological well-being. Another possibility is that the instrument used to measure mindfulness in this study is of a too global nature as mindfulness has been conceptualised as a multidimensional construct [37]. Perhaps specific mindfulness skills, such as accepting one's thoughts and feelings without judgement or having a nonreactive stance towards these internal phenomena may be more directly associated with various aspects of psychological well-being [37] and with type $\mathrm{D}$ personality than a general mindfulness measure.

One may speculate if an intervention aimed at the enhancement of specific mindfulness skills may be useful to increase psychological well-being in type D patients, as such mindfulness interventions have been found to enhance psychological well-being, satisfaction with life and coping with pain in various patient populations [23-26, 28, 29]. Alternatively, it has been suggested that more patient-tailored interventions may be needed for type $\mathrm{D}$ patients to benefit from psychological interventions [38]. Dependent on the specific problems and needs of an individual patient, such an intervention may apply a combination of approaches aimed at enhancing coping skills related to either the negative affectivity or social inhibition component of type D, or both. Such approaches may include cognitive-behavioural therapy or mindfulness-based cognitive therapy for the enhancement of mood and interpersonal therapy and assertiveness training for feeling more comfortable in social situations, including the context of interaction with health care providers [38]. Because such specific interventions for type $\mathrm{D}$ patients do not exist yet, despite the fact that this personality type is associated with decreased psychological well-being and satisfaction with life, the clinician is advised to (a) routinely screen for type D personality using the simple and brief 14-item DS-14 questionnaire [15] and (b) offer these patients the possibility for a conversation with a psychologist, who may assess in more depth the risk of psychological problems in these patients, and possibly offer them specific psychological treatment.

This study has a number of limitations which must be considered when interpreting the results. First, the analyses involved correlational data gathered at one time point, precluding any possible conclusions along causal lines. Second, missing values in the disease activity score, especially regarding the erythrocyte sedimentation, resulted in reduced power of analyses on this outcome. In addition, it was not possible to obtain erythrocyte scores at the same time point as the other data, introducing a small time gap between the assessment times, being 5.5 days on average, potentially decreasing the effect size.

A merit of the study is the high response rate. Ninety-one percent of patients asked to participate completed the questionnaires, resulting in adequate generalisability of the present results to the RA population from which the participants were drawn.

In conclusion, the present study showed associations of the distressed (type D) personality with subjective indices of aspects of quality of life, such as psychological well-being and life satisfaction in RA patients, but not with disability or objective indices of disease activity. Future research including prospective follow-up assessments is needed to be able to help to draw conclusions along causal lines. If the present results are confirmed and extended in future studies, interventions may be designed to meet the needs of patients with a type $\mathrm{D}$ personality, as outlined above.

Acknowledgements The authors would like to thank Dr. Eveline Bicknese, Dr. Frans Hoogwegt and Dr. Anton Westgeest of Máxima Medical Center Eindhoven for their cooperation in providing the context necessary for conducting the present study.

\section{Disclosures None.}

Open Access This article is distributed under the terms of the Creative Commons Attribution Noncommercial License which permits any noncommercial use, distribution, and reproduction in any medium, provided the original author(s) and source are credited.

\section{References}

1. Symmons D, Turner G, Webb R, Asten P, Barrett E, Lunt M, Scott D, Silman A (2002) The prevalence of rheumatoid arthritis in the United Kingdom: new estimates for a new century. Rheumatology (Oxford) 41:793-800

2. van Riel PL, van Gestel AM, van de Putte LB (1996) Development and validation of response criteria in rheumatoid arthritis: steps towards an international consensus on prognostic markers. Br J Rheumatol 35(Suppl 2):4-7

3. Krol B (1996) Quality of life in rheumatoid arthritis patients: the relation between personality, social support and depression [unpublished dissertation]. Groningen University, Groningen

4. Pincus T, Griffith J, Pearce S, Isenberg D (1996) Prevalence of self-reported depression in patients with rheumatoid arthritis. Br J Rheumatol 35:879-883

5. Chou CY, Brauer DJ (2005) Temperament and satisfaction with health status among persons with rheumatoid arthritis. Clin Nurse Spec 19:94-100 
6. Curtis R, Groarke A, Coughlan R, Gsel A (2005) Psychological stress as a predictor of psychological adjustment and health status in patients with rheumatoid arthritis. Patient Educ Couns 59:192-198

7. Denollet J, Vaes J, Brutsaert DL (2000) Inadequate response to treatment in coronary heart disease: adverse effects of type D personality and younger age on 5-year prognosis and quality of life. Circulation 102:630-635

8. Treharne GJ, Lyons AC, Booth DA, Kitas GD (2007) Psychological well-being across 1 year with rheumatoid arthritis: coping resources as buffers of perceived stress. Br J Health Psychol 12:323-345

9. Andersson G (1996) The benefits of optimism: a meta-analytic review of the Life Orientation Test. Pers Indiv Diff 21:719-725

10. Hagglund KJ, Haley WE, Reveille JD, Alarcon GS (1989) Predicting individual differences in pain and functional impairment among patients with rheumatoid arthritis. Arthritis Rheum 32:851-858

11. Suurmeijer TPBM, Van Sonderen FLP, Krol B, Doeglas DM, Van Den Heuvel WJA, Sanderman R (2005) The relationship between personality, supportive transactions and support satisfaction, and mental health of patients with early rheumatoid arthritis. Results from the Dutch part of the EURIDISS study. Soc Indicat Res 73:179-197

12. Watson D, Pennebaker JW (1989) Health complaints, stress, and distress: exploring the central role of negative affectivity. Psychol Rev 96:234-254

13. Mols F, Denollet J (2010) Type D personality among noncardiovascular patient populations: a systematic review. Gen Hosp Psychiatry 32:66-72

14. Denollet J, Sys SU, Stroobant N, Rombouts H, Gillebert TC, Brutsaert DL (1996) Personality as independent predictor of long-term mortality in patients with coronary heart disease. Lancet 347:417-421

15. Denollet J (2005) DS14: standard assessment of negative affectivity, social inhibition, and type D personality. Psychosom Med 67:89-97

16. Pedersen SS, Denollet J (2006) Is type D personality here to stay? Emerging evidence across cardiovascular disease patient groups. Curr Cardiol Rev 2:205-213

17. Schiffer AA, Pedersen SS, Widdershoven JW, Hendriks EH, Winter JB, Denollet J (2005) The distressed (type D) personality is independently associated with impaired health status and increased depressive symptoms in chronic heart failure. Eur J Cardiovasc Prev Rehabil 12:341-346

18. Pedersen SS, Denollet J (2003) Type D personality, cardiac events, and impaired quality of life: a review. Eur J Cardiovasc Prev Rehabil 10:241-248

19. Denollet J, Pedersen SS, Vrints CJ, Conraads VM (2006) Usefulness of type D personality in predicting five-year cardiac events above and beyond concurrent symptoms of stress in patients with coronary heart disease. Am J Cardiol 97:970-973

20. Denollet J, Conraads VM, Brutsaert DL, De Clerck LS, Stevens WJ, Vrints CJ (2003) Cytokines and immune activation in systolic heart failure: the role of type D personality. Brain Behav Immun 17:304-309

21. Ackermann C, Kavanaugh A (2007) Tumor necrosis factor as a therapeutic target of rheumatologic disease. Expert Opin Ther Targets 11:1369-1384

22. Blackledge JT, Hayes SC (2001) Emotion regulation in acceptance and commitment therapy. J Clin Psychol 57:243-255
23. Carlson LE, Speca M, Patel KD, Goodey E (2003) Mindfulnessbased stress reduction in relation to quality of life, mood, symptoms of stress, and immune parameters in breast and prostate cancer outpatients. Psychosom Med 65:571-581

24. Nyklíček I, Kuijpers KF (2008) Effects of mindfulness-based stress reduction intervention on psychological well-being and quality of life: is increased mindfulness indeed the mechanism? Ann Behav Med 35:331-340

25. Witek-Janusek L, Albuquerque K, Chroniak KR, Chroniak C, Durazo-Arvizu R, Mathews HL (2008) Effect of mindfulness based stress reduction on immune function, quality of life and coping in women newly diagnosed with early stage breast cancer. Brain Behav Immun 22:969-981

26. Grossman P, Tiefenthaler-Gilmer U, Raysz A, Kesper U (2007) Mindfulness training as an intervention for fibromyalgia: evidence of postintervention and 3-year follow-up benefits in well-being. Psychother Psychosom 76:226-233

27. Kingston J, Chadwick P, Meron D, Skinner TC (2007) A pilot randomized control trial investigating the effect of mindfulness practice on pain tolerance, psychological well-being, and physiological activity. J Psychosom Res 62:297-300

28. Veehof MM, Ten Klooster PM, Taal E, Westerhof GJ, Bohlmeijer ET (2011) Psychometric properties of the Dutch Five Facet Mindfulness Questionnaire (FFMQ) in patients with fibromyalgia. Clin Rheumatol 30:1045-1054

29. Carlson LE, Speca M, Patel KD, Faris P (2007) One year pre-post intervention follow-up of psychological, immune, endocrine and blood pressure outcomes of mindfulness-based stress reduction (MBSR) in breast and prostate cancer outpatients. Brain Behav Immun 21:1038-1049

30. Walach H, Buchheld N, Buttenmüller V, Kleinknecht N, Schmidt S (2006) Measuring mindfulness: the Freiburg Mindfulness Inventory. Pers Indiv Diff 40:1543-1555

31. Bruce B, Fries JF (2003) The Stanford Health Assessment Questionnaire: a review of its history, issues, progress, and documentation. J Rheumatol 30:167-178

32. Boers M, Jacobs JW, van Vliet Vlieland TP, van Riel PL (2007) Consensus Dutch health assessment questionnaire. Ann Rheum Dis 66:132-133

33. Arrindell WA, Meeuwesen L, Huyse FJ (1991) The Satisfaction with Life Scale (SWLS): psychometric properties in a non-psychiatric medical outpatients sample. Pers Indiv Diff 12:117-123

34. Denollet J (1993) Emotional distress and fatigue in coronary heart disease: the Global Mood Scale (GMS). Psychol Med 23:111-121

35. Denollet J (2006) Recognizing increased risk of depressive comorbidity after myocardial infarction: looking for 4 symptoms of anxiety-depression. Psychother Psychosom 75:346-352

36. Prevoo ML, van 't Hof MA, Kuper HH, van Leeuwen MA, van de Putte LB, van Riel PL (1995) Modified disease activity scores that include twenty-eight-joint counts. Development and validation in a prospective longitudinal study of patients with rheumatoid arthritis. Arthritis Rheum 38:44-48

37. Baer RA, Smith GT, Hopkins J, Krietemeyer J, Toney L (2006) Using self-report assessment methods to explore facets of mindfulness. Assessment 13:27-45

38. Pelle AJ, Van den Broek KC, Denollet J (in press) Interventions in the context of the distressed (type D) personality. In: Dornelas AE (ed). Stress proof the heart. Springer, New York 